KSUPT-11/5 October 2011

\title{
Constraints on dark energy from H II starburst galaxy apparent magnitude versus redshift data
}

\author{
Data Mania ${ }^{1,2}$ and Bharat Ratra ${ }^{1}$
}

\begin{abstract}
In this paper we use H II starburst galaxy apparent magnitude versus redshift data from Siegel et al. (2005) to constrain dark energy cosmological model parameters. These constraints are generally consistent with those derived using other data sets, but are not as restrictive as the tightest currently available constraints.
\end{abstract}

\section{Introduction}

There is significant observational evidence supporting the idea that we live in a spatiallyflat Universe that is currently undergoing accelerated cosmological expansion. Most cosmologists believe that this accelerated expansion is powered by dark energy which dominates the current cosmological energy budget (for reviews of dark energy see Tsujikawa 2010; Linder 2010; Blanchard 2010; Sapone 2010, and references therein) 3

The energy budget of the "standard" model of cosmology — the spatially-flat $\Lambda$ CDM model (Peebles 1984) — is currently dominated by far by dark energy: Einstein's cosmological constant $\Lambda$ contributes more than $70 \%$ of the budget. Nonrelativistic cold dark matter (CDM) is the next largest contributor (more than $20 \%$ ), followed by nonrelativistic baryons

\footnotetext{
${ }^{1}$ Department of Physics, Kansas State University, 116 Cardwell Hall, Manhattan, KS 66506, USA mania, ratra@phys.ksu.edu

${ }^{2}$ Center for Elementary Particle Physics, Ilia State University, 3-5 Cholokashvili Ave., Tbilisi 0179, Georgia

3 Some, however, feel that this accelerated expansion is better viewed as an indication that the general relativistic description of gravitation needs to be improved on (see de Felice \& Tsujikawa 2010; Tsujikawa 2010; Jain \& Khourv 2010, and references therein). In this paper we assume that general relativity provides an adequate description of gravitation on cosmological scales.
} 
(around $5 \%$ ). For a review of the standard model see Ratra \& Vogelev (2008) and references therein. It has been know for some time that the $\Lambda$ CDM model is reasonably consistent with most observational constraints (see, e.g., Jassal et al. 2010; Wilson et al. 2006; Davis et al. 2007; Allen et al. 2008, for early indications) 4

However, the $\Lambda$ CDM model appears to leave some conceptual questions unanswered. One of these is that the naive energy density scale we expect from quantum field theory considerations is many orders of magnitude higher than the measured cosmological constant energy density scale. Another is what is known as the coincidence puzzle: The energy density of a cosmological constant does not change over time but the matter density decreases with the cosmological expansion, so it is puzzle why we observers live at this seemingly special time, when the dark energy and nonrelativistic matter densities happen to be of comparable size.

These puzzles, and perhaps others, could be alleviated if we assume that the dark energy density was higher in the past and slowly decreased in time, thus remaining comparable to the nonrelativistic matter density for a longer time (Ratra \& Peebles 1988). Many such timevarying dark energy models have been proposed 5 In this paper, for illustrative purposes, we consider two dark energy models and one dark energy parametrization.

In the $\Lambda \mathrm{CDM}$ model, time-independent dark energy — the cosmological constant — may be modeled as a spatially homogeneous fluid with equation of state parameter $\omega_{\Lambda}=$ $p_{\Lambda} / \rho_{\Lambda}=-1$ (where $p_{\Lambda}$ and $\rho_{\Lambda}$ are the fluid pressure and energy density).

In an attempt to describe slowly decreasing dark energy, the dark energy fluid equation of state is extended to the XCDM $X$-fluid parametrization. Here, dark energy is again modeled as a spatially homogeneous $(X)$ fluid, but now with an equation of state parameter $w_{X}=p_{X} / \rho_{X}$, where $w_{X}(<-1 / 3)$ is an arbitrary constant and $p_{X}$ and $\rho_{X}$ are the pressure and energy density of the $X$-fluid. When $w_{X}=-1$ the XCDM parametrization reduces to the complete and consistent $\Lambda \mathrm{CDM}$ model. For any other value of $w_{X}(<-1 / 3)$, the XCDM parametrization is incomplete as it cannot describe spatial inhomogeneities (see, e.g. Ratra 1991; Podariu \& Ratra 2000). For computational simplicity, here we study the XCDM parametrization in only the spatially-flat cosmological case.

A slowly rolling scalar field $\phi$, with decreasing (in $\phi$ ) potential energy density $V(\phi)$, can

\footnotetext{
${ }^{4}$ The $\Lambda$ CDM model assumes the "standard" CDM structure formation picture, which might be in some observational difficulty (see, e.g., Peebles \& Ratra 2003; Perivolaropoulos 2010).

${ }^{5}$ For recent discussions see, e.g., Novosvadlyi et al. (2010), Harko \& Lobo (2010), Keresztes et al. (2010), Pettorino et al. (2010), Liu et al. (2010), Costa (2010), Farajollahi et al. (2011), and references therein.
} 
be used to consistently model a gradually decreasing, time-evolving, dark energy density. An inverse power-law potential energy density $V(\phi) \propto \phi^{-\alpha}$, where $\alpha$ is a non-negative constant, provides a simple realization of this $\phi$ CDM scenario (Peebles \& Ratra 1988; Ratra \& Peebles 1988). When $\alpha=0$ the $\phi \mathrm{CDM}$ model reduces to the corresponding $\Lambda$ CDM case. For computational simplicity, here we study the $\phi \mathrm{CDM}$ model in only the spatially-flat cosmological case.

Current observational data convincingly indicate that the cosmological expansion is accelerating. The main support for accelerated expansion comes from three types of data: supernova Type Ia (SNIa) apparent magnitude versus redshift measurements (see, e.g., Amanullah et al. 2010; Holsclaw et al. 2010; Wang et al. 2011; Guv et al. 2010); cosmic microwave background (CMB) anisotropy data (see, e.g., Podariu et al. 2001b; Komatsu et al. 2009; Liu et al. 2011; La Vacca \& Bonometto 2011) combined with low estimates of the cosmological matter density (see, e.g, Chen \& Ratra 2003b); and, baryon acoustic oscillation (BAO) peak length scale estimates (see, e.g., Samushia \& Ratra 2009; Wang 2010; Percival et al. 2010; Blake et al. 2011). However, the error bars associated with these three kinds of data are still too large to allow for a significant observational discrimination between the $\Lambda$ CDM model and the two simple time-varying dark energy models discussed above.

There are two major reasons to consider additional data sets. First, it is of great interest to check the above results by comparing to results derived from other data. If the new constraints differ significantly from the old ones, this could mean that the model being tested is observationally inconsistent, or it could mean that one of the data sets had an undetected systematic error. Either of these is a significant result. On the other hand, if the constraints from the new and the old data are consistent, then a joint analysis of all the data could result in significantly tighter constraints, and so possibly result in significantly discriminating between constant and time-varying dark energy.

Other data that have recently been used to constrain dark energy models include strong gravitational lensing measurements (e.g., Chae et al. 2004; Lee \& Ng 2007; Zhang \& Wu 2010; Biesiada et al. 2010), angular size as a function of redshift observations (e.g., Guerra et al. 2000; Chen \& Ratra 2003a; Bonamente et al. 2006), Hubble parameter as a function of redshift measurements (e.g., Jimenez et al. 2003; Samushia \& Ratra 2006; Sen \& Scherrer 2008; Samushia et al. 2007; Pan et al. 2010), galaxy cluster gas mass fraction data (e.g., Allen et al. 2008; Samushia \& Ratra 2008; Ettori et al. 2009; Tong \& Noh 2011), and large-scale structure observations (e.g., Baldi \& Pettorino 2011; De Boni et al. 2011; Mortonson et al. 2011; Brouzakis et al. 2010; Campanelli et al. 2011).

While constraints from these data are less restrictive than those derived from the SNeIa, $\mathrm{CMB}$ and $\mathrm{BAO}$ data, both types of data result in largely compatible parameter restrictions 
that generally support a currently accelerating cosmological expansion. This gives us confidence that the broad outlines of the "standard" cosmological model are now in place. However, there is still ambiguity. Current observations are unable to differentiate between different dark energy models. For instance, while current data favor a time-independent cosmological constant, they are unable to rule out time-varying dark energy. More and higher-quality data is needed to accomplish this important task.

It is anticipated that future space missions will result in significantly more and better SNeIa, BAO, and CMB anisotropy data (see, e.g., Podariu et al. 2001a; Samushia et al. 2011; Wang et al. 2010; Astier et al. 2010). A complementary approach is to develop cosmological tests that make use of different sets of objects. Recent examples include the lookback time test (e.g., Pires et al. 2006; Dantas et al. 2011) and the gamma-ray burst luminosity versus redshft test (see, e.g. Schaefer 2007; Wang 2008; Xu \& Wang 2010). Gamma-ray bursts, in particular, are very luminous and can be seen to much higher redshift than the SNeIa and so probe an earlier cosmological epoch. H II starburst galaxies are also very luminous and can be seen to redshifts exceeding 3. Recent work has indicated that H II starbusrt galaxies might be standardizable candles (Melnick et al. 2000; Terlevich et al. 2002; Melnick 2003), because of the correlation between their velocity dispersion, $H_{\beta}$ luminosity, and metallicity (Melnick 1978; Terlevich \& Melnick 1981; Melnick et al. 1988).

In this paper we use H II galaxy data from Siegel et al. (2005) to constrain parameters of the three dark energy models mentioned above. Plionis et al. (2009, 2010, 2011) have used the Siegel et al. (2005) data to constrain the XCDM parametrization. Here we also constrain parameters of the consistent and physically motivated $\Lambda \mathrm{CDM}$ and $\phi \mathrm{CDM}$ cosmological models. We also derive constraints on the parameters of these models and the XCDM parametrization from a joint analysis of the Siegel et al. (2005) H II galaxy data and the Percival et al. (2010) BAO peak length scale measurements.

In the next section we list the basic equations for the dark energy models we consider. In Sec. 3 we discuss the analysis method used for the H II galaxy data and the resulting constraints and how they compare with those from other cosmological tests. Section 4 presents constraints from a joint analysis of H II and BAO data. Section 5 summarizes our results.

\section{Dark energy model equations}

The Friedman equation for the $\Lambda$ CDM model with spatial curvature is

$$
H\left(z, H_{0}, \mathbf{p}\right)=H_{0} \sqrt{\Omega_{m}(1+z)^{3}+\Omega_{\Lambda}+\Omega_{R}(1+z)^{2}} .
$$


Here $H_{0}$ is the Hubble constant and $H(z)$ is the Hubble parameter at redshift $z, \Omega_{m}, \Omega_{\Lambda}$, and $\Omega_{R}=1-\Omega_{m}-\Omega_{\Lambda}$ are the nonrelativistic (baryonic and cold dark) matter, cosmological constant, and space curvature energy density parameters, respectively, and the model parameter set $\mathbf{p}=\left(\Omega_{m}, \Omega_{\Lambda}\right)$.

For the spatially-flat XCDM parametrization, the Hubble parameter is

$$
H\left(z, H_{0}, \mathbf{p}\right)=H_{0} \sqrt{\Omega_{m}(1+z)^{3}+\left(1-\Omega_{m}\right)(1+z)^{3\left(1+w_{X}\right)}} .
$$

Here $w_{X}<-1 / 3$ and the model parameter set $\mathbf{p}=\left(\Omega_{m}, \omega_{X}\right)$.

For the spatially-flat $\phi \mathrm{CDM}$ case we have a coupled set of equations governing the scalar field and Hubble parameter evolution

$$
\begin{gathered}
\ddot{\phi}+3 H \dot{\phi}-\frac{\kappa \alpha}{2 G} \phi^{-(\alpha+1)}=0, \\
H\left(z, H_{0}, \mathbf{p}\right)=H_{0} \sqrt{\Omega_{m}(1+z)^{3}+\Omega_{\phi}(z)} .
\end{gathered}
$$

Here we consider the inverse power law scalar field $(\phi)$ potential energy density (Peebles \& Ratra 1988) so

$$
\Omega_{\phi}(z)=\frac{1}{12 H_{0}^{2}}\left(\dot{\phi}^{2}+\frac{\kappa}{G} \phi^{-\alpha}\right),
$$

where $G$ is the Newtonian gravitational constant and $\alpha>0$ is a free parameter (that determines $\kappa)$. In this case the model parameter set is $\mathbf{p}=\left(\Omega_{m}, \alpha\right)$.

The distance modulus as a function of redshift is

$$
\mu\left(z, H_{0}, \mathbf{p}\right)=25+5 \log [3000(1+z) y(z)]-5 \log h,
$$

where $h$ is the dimensionless Hubble constant defined from $H_{0}=100 h \mathrm{~km} \mathrm{~s}^{-1} \mathrm{Mpc}^{-1}$. In the spatially open case $y(z)$ is

$$
y(z, \mathbf{p})=\frac{1}{\sqrt{\Omega_{R}}} \sinh \left(\sqrt{\Omega_{R}} \int_{0}^{z} \frac{d z^{\prime}}{E\left(z^{\prime}\right)}\right),
$$

where $E(z)=H(z) / H_{0}$. When $\Omega_{R}=0$ this reduces to $y(z)=\int_{0}^{z} d z^{\prime} / E\left(z^{\prime}\right)$. The angular diameter distance $d_{A}=c y(z) /\left[H_{0}(1+z)\right]$. 


\section{Constraints from H II galaxy apparent magnitude data}

We use the $13 \mu_{\text {obs }}\left(z_{i}\right)$ measurements of (Siegel et al. 2005), listed in Table 1, to constrain cosmological parameters by minimizing

$$
\chi_{\mathrm{HII}}^{2}\left(H_{0}, \mathrm{p}\right)=\sum_{i=1}^{13} \frac{\left[\mu_{\mathrm{obs}}\left(z_{i}\right)-\mu_{\mathrm{pred}}\left(z_{i}, H_{0}, \mathbf{p}\right)\right]^{2}}{\sigma_{i}^{2}} .
$$

Here $z_{i}$ is the redshift at which $\mu_{\mathrm{obs}}\left(z_{i}\right)$ is measured, $\mu_{\text {pred }}\left(z_{i}, H_{0}, \mathbf{p}\right)$ is the predicted distance modulus at the same redshift for the model under consideration, and $\sigma_{i}$ is the average of the upper and lower error bars listed in Table 1.

The Siegel et al. (2005) measurements listed in Table 1 are computed from

$$
\mu_{\mathrm{obs}}=2.5 \log \left(\frac{\sigma^{5}}{F_{H \beta}}\right)-2.5 \log \left(\frac{O}{H}\right)-A_{H \beta}+Z_{0}
$$

where $F_{H \beta}$ and $A_{H \beta}$ are the $H_{\beta}$ flux and extinction and $O / H$ is a metallicity. Following Plionis et al. (2011), for the zero point magnitude we use $Z_{0}=-26.60$, use Hubble constant value $H_{0}=73 \mathrm{~km} \mathrm{~s}^{-1} \mathrm{Mpc}^{-1}$ (and do not account for the associated uncertainty), and also exclude two H II galaxies (Q1700-MD103 and SSA22a-MD41) that show signs of a considerable rotational velocity component (Erb et al. 2006).

The best fit parameter set $\mathbf{p}_{*}$ is where $\chi_{\mathrm{HII}}^{2}$ is at a minimum, $\chi_{\min }^{2}$. The $1 \sigma, 2 \sigma$, and $3 \sigma$ confidence intervals, in the two-dimensional parameter space, are enclosed by the contours where $\chi^{2}=\chi_{\min }^{2}+\Delta \chi^{2}$ with $\Delta \chi^{2}=2.30, \Delta \chi^{2}=6.17$, and $\Delta \chi^{2}=11.8$, respectively.

The H II galaxy data constraints on cosmological parameters are shown in Figs. 13. Our results generally agree with Plionis et al. (2011) for the XCDM parametrization (compare our Fig. 2 and their Fig. 10). The small differences are due to the fact that in our analysis we do not use specially weighted sigmas, but rather average distance moduli uncertainties, and also ignore gravitational lensing effects. Both analyses ignore small $H_{0}$ uncertainties; these are not important for our illustrative purposes here, but should be accounted for in an analysis of improved near-future H II galaxy data.

The H II galaxy data constraints in Figs. 1-3 are not as restrictive as those that follow from SNeIa, BAO, or CMB anistropy data. They are, however, comparable to those from Hubble parameter observations (see Chen \& Ratra 2011b, and references therein) or lookback time observations (see Samushia et al. 2010, and references therein), and somewhat more restrictive than angular diameter distance constraints (see Chen \& Ratra 2011a, and references therein) and gamma-ray burst luminosity distance ones (see Samushia \& Ratra 2010, and references therein). We again note that we have not accounted for the uncertainty 
in $H_{0}$ in our analysis, so the $\mathrm{H}$ II galaxy constraints derived here are a little more restrictive than they really are. However, our analysis clearly illustrates the potential constraining power of near-future H II galaxy luminosity distance data.

\section{Joint constraints from BAO and H II galaxy data}

To constrain observables using BAO data we follow the procedure of Percival et al. (2010). With $D_{V}(z)=\left[(1+z)^{2} d_{A}^{2} c z / H(z)\right]^{1 / 3}$, Percival et al. (2010) measure

$$
D_{V}(0.275)=(1104 \pm 30)\left(\frac{\Omega_{b} h^{2}}{0.02273}\right)^{-0.134}\left(\frac{\Omega_{m} h^{2}}{0.1326}\right)^{-0.255} \text { Mpc. }
$$

We use this to build the likelihood estimator $\mathcal{L}_{\mathrm{BAO}}$ with a Gaussian prior of $\Omega_{m} h^{2}=0.1326 \pm$ 0.0063 , and neglect the error for $\Omega_{b} h^{2}$ as WMAP5 data constrains it to $0.5 \%$ (Komatsu et al. 2009). The BAO data constraint contours are shown in Figs. 4-6.

To derive joint $\mathrm{H}$ II galaxy and $\mathrm{BAO}$ data constraints we maximize the products of likelihoods $\mathcal{L}(\mathbf{p})=\mathcal{L}_{\mathrm{HII}} \mathcal{L}_{\mathrm{BAO}}$, where $\mathcal{L}_{\mathrm{HII}}=e^{-\chi_{\mathrm{HI}}^{2} / 2}$, to get the best fit set of parameters $\mathbf{p}_{*}$, and the 1,2 , and $3 \sigma$ contours defined as points where the likelihood equals $e^{-2.30 / 2}, e^{-6.17 / 2}$, and $e^{-11.8 / 2}$, respectively, of the maximum likelihood value. These contours are shown in Figs. 4 - 6. Clearly, even adding currently available H II galaxy data to the mix tightens the constraints.

\section{Conclusion}

We have used the starburst galaxy luminosity distance data of Siegel et al. (2005) to constrain cosmological parameters in some dark energy models. The resulting constraints are largely consistent with those that follow from other currently available data although they are not as restrictive as the constraints derived from SNeIa, BAO, and CMB anisotropy data.

The Siegel et al. (2005) H II data are preliminary H II data. It is gratifying that they result in interesting constraints on cosmological parameters. We anticipate that new, nearfuture, H II galaxy data will provide very useful constraints on cosmological parameters that will complement those derived using other data.

DM thanks Manolis Plionis, Glenn Horton-Smith, and Yun Chen for valuable suggestions. We acknowledge support from DOE grant DEFG030-99EP41093, NSF grant AST- 
1109275, and from the SNSF (SCOPES grant No 128040).

\section{REFERENCES}

Allen, S. W., et al. 2008, MNRAS, 383, 879

Amanullah, R., et al. 2010, ApJ, 716, 712

Astier, P., et al. 2010, arXiv:1010.0509 [astro-ph.CO]

Baldi, M., \& Pettorino, V. 2011, MNRAS, 412, L1

Biesiada, M., Piórkowska, A., \& Malec, B. 2010, MNRAS, 406, 1055

Blake, C., et al. 2011, arXiv:1108.2635 [astro-ph.CO]

Blanchard, A. 2010, A\&A Rev., 18, 595

Bonamente, M., et al. 2006, ApJ, 647, 25

Brouzakis, N., et al. 2011, J. Cosmology Astropart. Phys., 1103, 049

Campanelli, L., et al. 2011, arXiv:1110.2310 [astro-ph.CO]

Chae, K.-H., et al. 2004, ApJ, 607, L71

Chen, G., \& Ratra, B. 2003a, ApJ, 582, 586

Chen, G., \& Ratra, B. 2003b, PASP, 115, 1143

Chen, Y., \& Ratra, B. 2011a, arXiv:1105.5660 [astro-ph.CO]

Chen, Y., \& Ratra, B. 2011b, Phys. Lett. B, 703, 406

Costa, F. E. M. 2010, Phys. Rev. D, 82, 103527

Dantas, M. A., et al. 2011, Phys. Lett. B, 699, 239

Davis, T. M., et al. 2007, ApJ, 666, 716

De Boni, C., et al. 2011, MNRAS, 415, 2758

de Felice, A., \& Tsujikawa, S. 2010, Living Rev. Rel., 13, 3

Erb, D. K., et al. 2006, ApJ, 646, 107 
Ettori, S., et al. 2009, A\&A, 501, 61

Farajollahi, H., et al. 2011, Ap\&SS, 563

Guerra, E. J., Daly, R. A., \& Wan, L. 2000, ApJ, 544, 659

Guy, J., et al. 2010, A\&A, 523, A7

Harko, T., \& Lobo, F. S. N. 2010, Eur. Phys. J. C, 70, 373

Holsclaw, T., et al. 2010, Phys. Rev. Lett., 105, 241302

Jain, B., \& Khoury, J. 2010, Annals Phys., 325, 1479

Jassal, H. K., Bagla, J. S., \& Padmanabhan, T. 2010, MNRAS, 405, 2639

Jimenez, R., et al. 2003, ApJ, 593, 622

Keresztes, Z., et al. 2010, Phys. Rev. D, 82, 123534

Komatsu, E., et al. 2009, ApJS, 180, 330

La Vacca, G., \& Bonometto, S. A.. 2011, Nucl. Phys. Proc. Suppl., 217, 68

Lee, S., \& Ng, K.-W. 2007, Phys. Rev. D, 76, 043518

Linder, E. V. 2010, arXiv:1004.4646 [astro-ph.CO]

Liu, D.-J., Wang, H., \& Yang, B. 2010, Phys. Lett. B, 694, 6

Liu, J., Li, M., \& Zhang, X. 2011, J. Cosmology Astropart. Phys., 1106, 028

Melnick, J. 1978, A\&A, 70, 157

Melnick, J. 2003, ASP Conf. Proc. Ser., 297, 3

Melnick, J., Terlevich, R., \& Moles, M. 1988, MNRAS, 235, 297

Melnick, J., Terlevich, R., \& Terlevich, E. 2000, MNRAS, 311, 629

Mortonson, M. J., Hu, W., \& Huterer, D. 2011, Phys. Rev. D, 83, 023015

Novosyadlyj, B., et al. 2010, Phys. Rev. D, 82, 103008

Pan, N., et al. 2010, Class. Quantum Grav., 27, 155015

Peebles, P. J. E. 1984, ApJ, 284, 439 
Peebles, P. J. E., \& Ratra, B. 1988, ApJ, 325, L17

Peebles, P. J. E., \& Ratra, B. 2003, Rev. Mod. Phys., 75, 559

Percival, W. J., et al. 2010, MNRAS, 401, 2148

Perivolaropoulos, L. 2010, J. Phys. Conf. Ser., 222, 012024

Pettorino, V., et al. 2010, Phys. Rev. D, 82, 123001

Pires, N., Zhu, Z.-H., \& Alcaniz, J. S. 2006, Phys. Rev. D, 73, 123530

Plionis, M., et al. 2010, AIP Conf. Proc. 1241, 267

Plionis, M., et al. 2011, MNRAS, 416, 2981

Plionis, M., et al. 2009, J. Phys. Conf. Ser., 189, 012032

Podariu, S., Nugent, P., \& Ratra, B. 2001a, ApJ, 553, 39

Podariu, S., et al. 2001b, ApJ, 559, 9

Podariu, S., \& Ratra, B. 2000, ApJ, 532, 109

Ratra, B. 1991, Phys. Rev. D, 43, 3802

Ratra, B., \& Peebles, P. J. E. 1988, Phys. Rev. D, 37, 3406

Ratra, B., \& Vogeley, M. S. 2008, PASP, 120, 235

Samushia, L., Chen, G., \& Ratra, B. 2007, arXiv:0706.1963 [astro-ph]

Samushia, L., et al. 2010, Phys. Lett. B, 693, 509

Samushia, L., \& Ratra, B. 2006, ApJ, 650, L5

Samushia, L., \& Ratra, B. 2008, ApJ, 680, L1

Samushia, L., \& Ratra, B. 2009, ApJ, 701, 1373

Samushia, L., \& Ratra, B. 2010, ApJ, 714, 1347

Samushia, L., et al. 2011, MNRAS, 410, 1993

Sanderson, C. 2010, Tech. rep., NICTA

Sapone, D. 2010, Int. J. Mod. Phys. A, 25, 5253 
Schaefer, B. E. 2007, ApJ, 660, 16

Sen, A. A., \& Scherrer, R. J. 2008, Phys. Lett. B, 659, 457

Siegel, E. R., et al. 2005, MNRAS, 356, 1117

Terlevich, R., \& Melnick, J. 1981, MNRAS, 195, 839

Terlevich, E., Terlevich, R., \& Melnick, J. 2002, Rev. Mex. A\&A (Ser. Conf.), 12, 272

Tong, M., \& Noh, H. 2011, Eur. Phys. J. C, 71, 1586

Tsujikawa, S. 2010, arXiv:1004.1493 [astro-ph.CO]

Wang, S., Li, X.-D., \& Li, M. 2011, Phys. Rev. D, 83, 023010

Wang, Y. 2008, Phys. Rev. D, 78, 123532

Wang, Y. 2010, Mod. Phys. Lett. A, 25, 3093

Wang, Y., et al. 2010, MNRAS, 409, 737

Wilson, K. M., Chen, G., \& Ratra, B. 2006, Mod. Phys. Lett. A, 21, 2197

Xu, L., \& Wang, Y. 2010, J. Cosmology Astropart. Phys., 1011, 014

Zhang, Q.-J., \& Wu, Y.-L. 2010, J. Cosmology Astropart. Phys., 1008, 038 


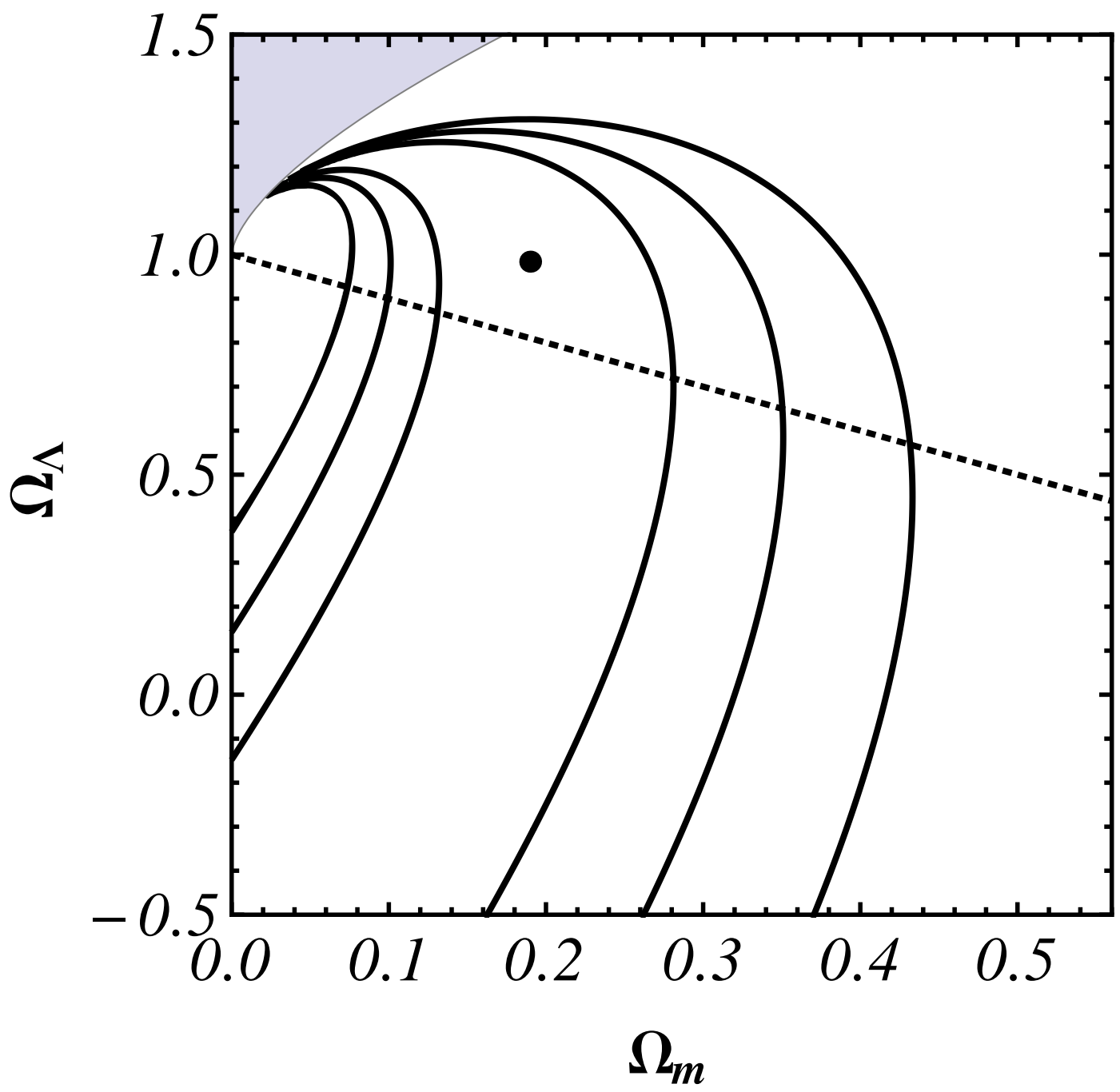

Fig. 1.- H II galaxy data 1,2 , and $3 \sigma$ confidence level contours in the $\left(\Omega_{m}, \Omega_{\Lambda}\right)$ plane for the $\Lambda \mathrm{CDM}$ model. The dotted line corresponds to the spatially-flat $\Lambda \mathrm{CDM}$ case and the shaded area in the upper left hand corner is the part of parameter space without a big bang. The best-fit point with $\chi_{\min }^{2}=53.3$ is indicated by the solid black circle at $\Omega_{m}=0.19$ and $\Omega_{\Lambda}=0.98$. 


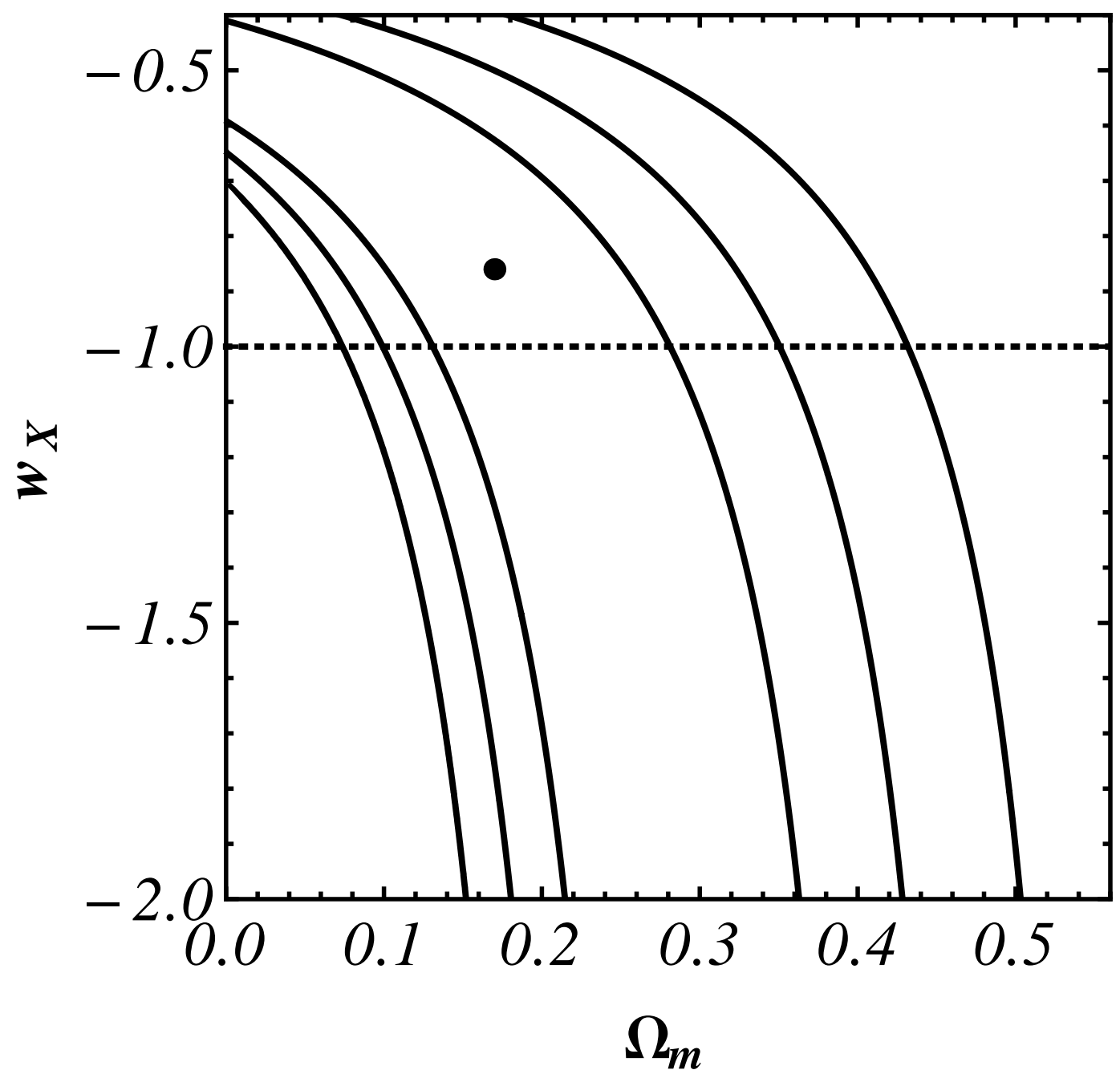

Fig. 2.- H II galaxy data 1,2 , and $3 \sigma$ confidence level contours in the $\left(\Omega_{m}, w_{X}\right)$ plane for the spatially-flat XCDM parametrization. The dotted line corresponds to the spatially-flat $\Lambda \mathrm{CDM}$ case. The best-fit point with $\chi_{\min }^{2}=53.3$ is indicated by the solid black circle at $\Omega_{m}=0.17$ and $w_{X}=-0.86$. 


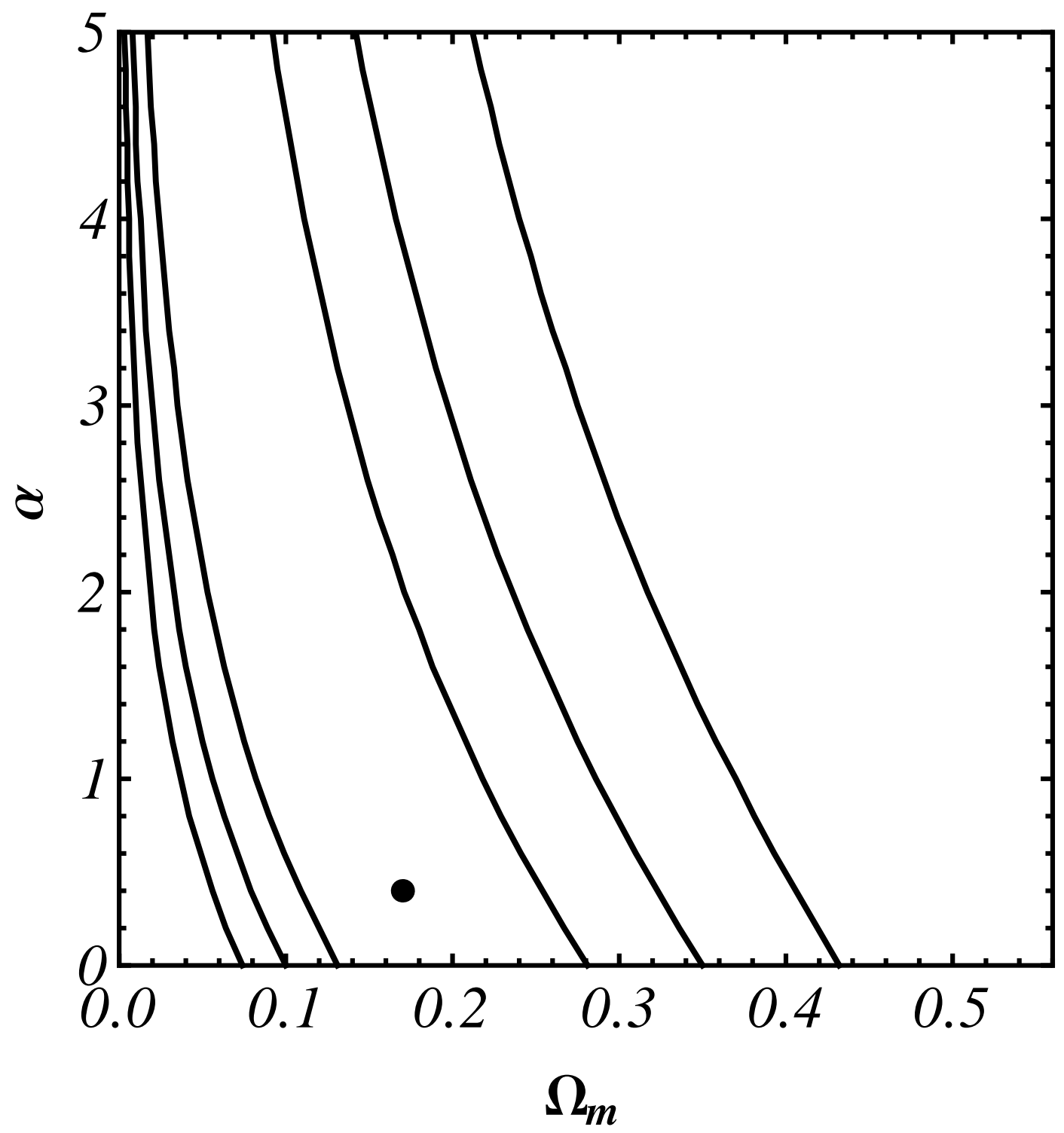

Fig. 3.- H II galaxy data 1,2 , and $3 \sigma$ confidence level contours in the $\left(\Omega_{m}, \alpha\right)$ plane for the spatially-flat $\phi \mathrm{CDM}$ model. $\alpha=0$ corresponds to the spatially-flat $\Lambda \mathrm{CDM}$ case. The best-fit point with $\chi_{\min }^{2}=53.3$ is indicated by the solid black circle at $\Omega_{m}=0.17$ and $\alpha=0.39$. 


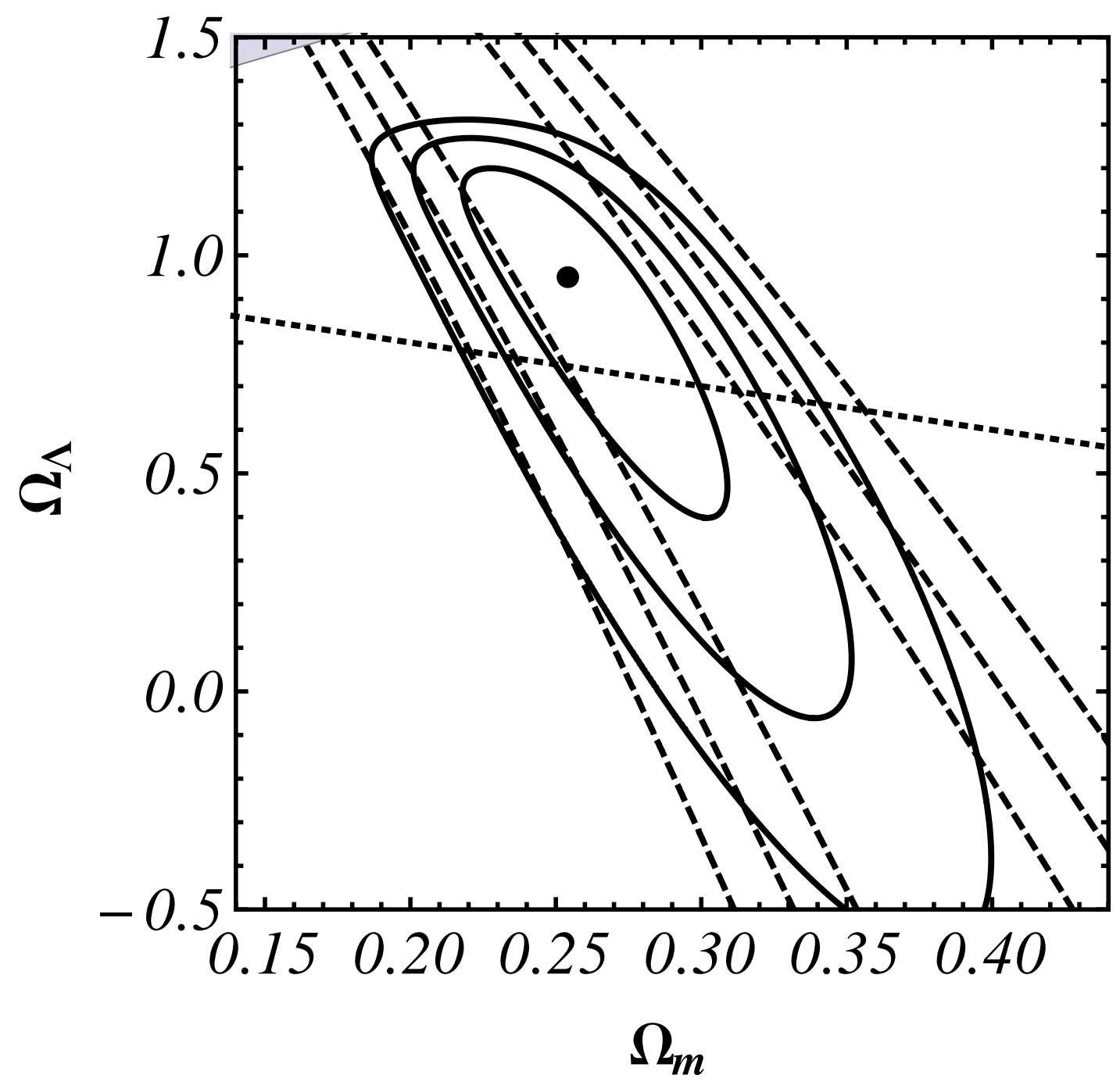

Fig. 4.- Joint H II galaxy and BAO data (solid lines) and BAO data only (dashed lines) 1, 2 , and $3 \sigma$ confidence level contours in the $\left(\Omega_{m}, \Omega_{\Lambda}\right)$ plane for the $\Lambda \mathrm{CDM}$ model. Conventions and notation are as in Fig. 1 . The best-fit point with $-2 \log \left(\mathcal{L}_{\max }\right)=55.2$ is indicated by the solid black circle at $\Omega_{m}=0.25$ and $\Omega_{\Lambda}=0.95$. 


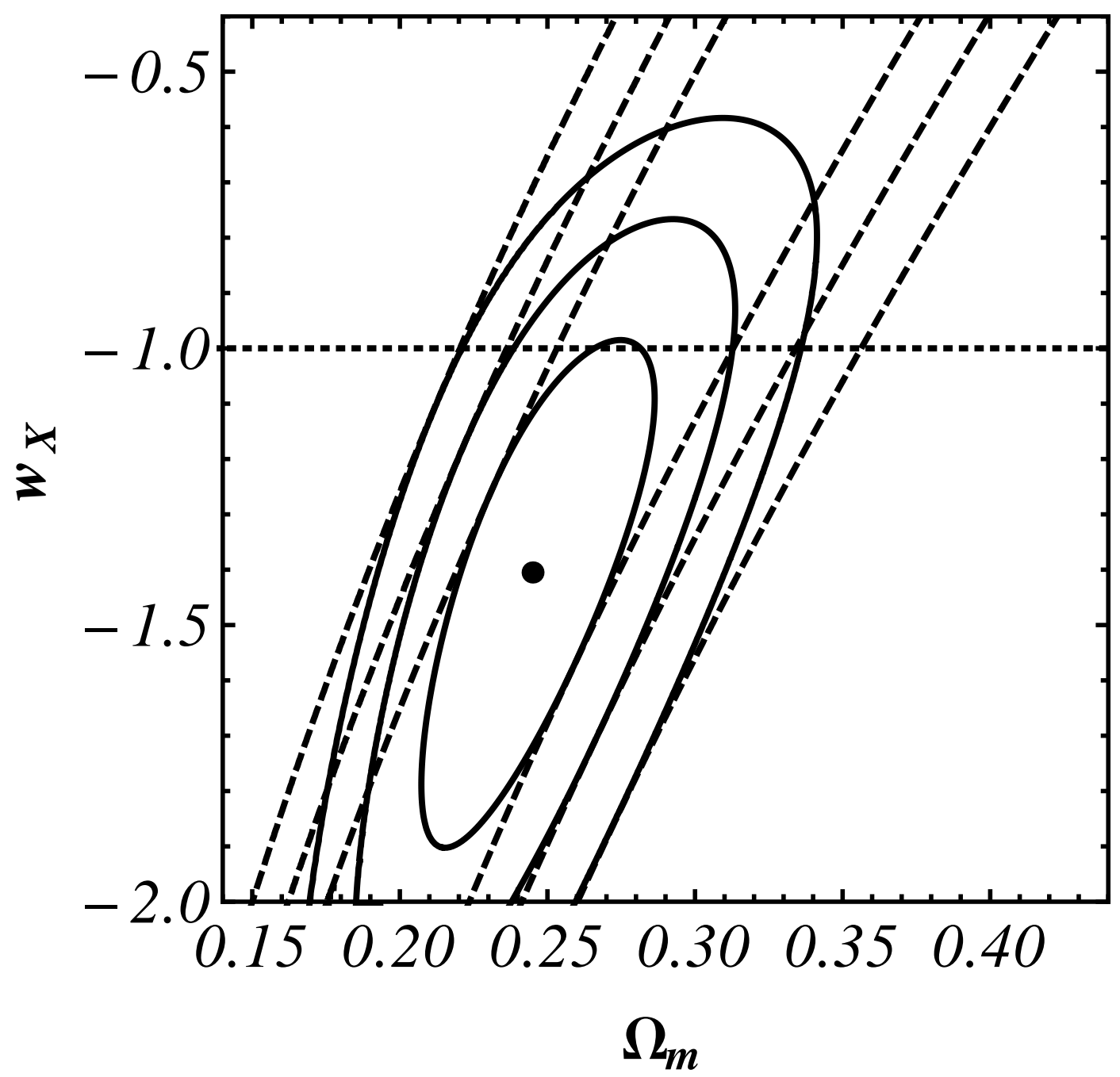

Fig. 5.- Joint H II galaxy and BAO data (solid lines) and BAO data only (dashed lines) 1,2 , and $3 \sigma$ confidence level contours in the $\left(\Omega_{m}, w_{X}\right)$ plane for the spatially-flat XCDM parametrization. Conventions and notation are as in Fig. 2. The best-fit point with $-2 \log \left(L_{\max }\right)=53.5$ is indicated by the solid black circle at $\Omega_{m}=0.25$ and $w_{x}=-1.41$. 


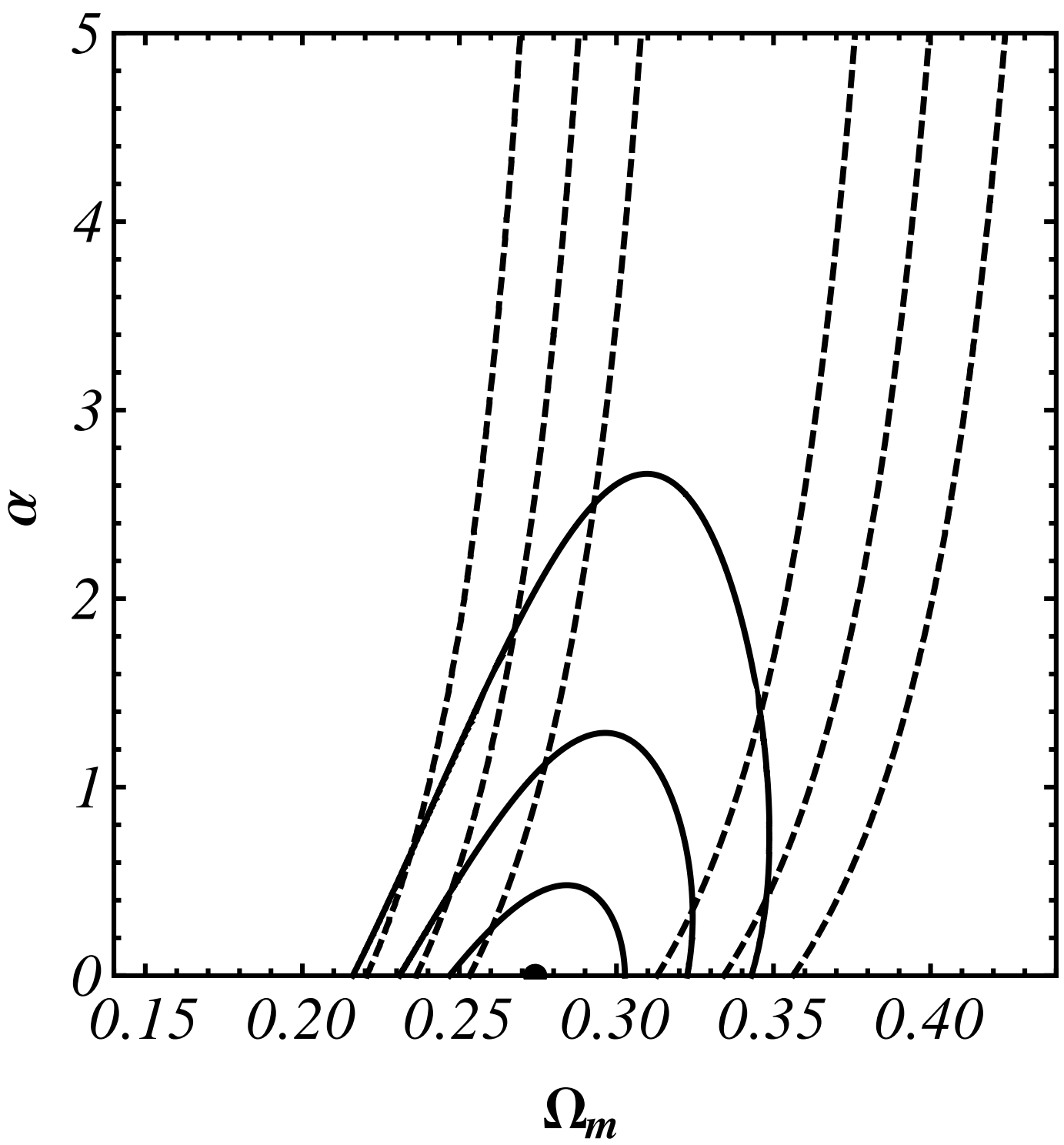

Fig. 6.- Joint H II galaxy and BAO data (solid lines) and BAO data only (dashed lines) 1, 2 , and $3 \sigma$ confidence level contours in the $\left(\Omega_{m}, \alpha\right)$ plane for the spatially-flat $\phi \mathrm{CDM}$ model Conventions and notation are as in Fig. 3. The best-fit point with $-2 \log \left(\mathcal{L}_{\max }\right)=55.6$ is indicated by the solid black circle at $\Omega_{m}=0.27$ and $\alpha=0$. 
Table 1: Siegel et al. (2005) H II starburst galaxy distance moduli and uncertainties

\begin{tabular}{l|c|c} 
Galaxy & $z$ & $\mu_{\mathrm{obs}} \pm \sigma$ \\
\hline \hline Q0201-B13 & 2.17 & $47.49_{-3.43}^{+2.10}$ \\
Q1623-BX432 & 2.18 & $45.45_{-3.07}^{+1.97}$ \\
Q1623-MD107 & 2.54 & $44.82_{-1.58}^{+0.31}$ \\
Q1700-BX717 & 2.44 & $46.64_{-1.58}^{+0.31}$ \\
CDFa C1 & 3.11 & $45.77_{-1.58}^{+0.31}$ \\
Q0347-383 C5 & 3.23 & $47.12_{-0.32}^{+0.32}$ \\
B2 0902+343 C12 & 3.39 & $46.96_{-0.81}^{+0.71}$ \\
Q1422+231 D81 & 3.10 & $48.81_{-0.40}^{+0.38}$ \\
SSA22a-MD46 & 3.09 & $46.76_{-0.51}^{+0.56}$ \\
SSA22a-D3 & 3.07 & $49.71_{-0.41}^{+0.43}$ \\
DSF2237+116a C2 & 3.32 & $47.73_{-0.25}^{+0.25}$ \\
B2 0902+343 C6 & 3.09 & $45.22_{-1.76}^{+1.38}$ \\
MS1512-CB58 & 2.73 & $47.49_{-1.57}^{+1.22}$
\end{tabular}

